\title{
Hydrogen Sorption in Functionalized Metal-Organic Frameworks (Supplementary Information)
}

\author{
Jesse L. C. Rowsell, Andrew R. Millward, Kyo Sung Park and Omar M. Yaghi* \\ Department of Chemistry, University of Michigan, 930 North University Avenue, Ann Arbor, Michigan, 48109, USA
}

\section{Synthesis of IRMOF-18, $\mathrm{Zn}_{4} \mathrm{O}(\mathrm{TMBDC})_{3}$}

2,3,5,6-tetramethylbenzene-1,4-dicarboxylic acid (1.2 g, $5.4 \mathrm{mmol}$, Chem Service) and zinc nitrate hexahydrate (12 g, $40 \mathrm{mmol}$, Aldrich) were dissolved in $600 \mathrm{~mL}$ of $\mathrm{N}, \mathrm{N}-$ diethylformamide (BASF) with stirring and distributed into six large glass crystallization dishes. The covered dishes were heated for $48 \mathrm{hr}$ at $70^{\circ} \mathrm{C}$, during which time a white polycrystalline solid formed on the surface of the vessel. After removal from the oven, the mother liquor was decanted and the solid was washed with $\mathrm{N}, \mathrm{N}$-dimethylformamide to remove unreacted material. The vessels were then filled with $100 \mathrm{~mL}$ of chloroform (Fisher), immersing the product. This solvent volume was replaced after $4 \mathrm{hr}$ and allowed to sit for 16 $\mathrm{hr}$ before replacing the solvent a second time. After immersion for another $24 \mathrm{hr}$, the solids were scraped off the surface of the vessel and filtered. The guest chloroform was removed under vacuum $\left(<10^{-3}\right.$ Torr $)$ to yield the porous material $(1.3 \mathrm{~g}, 77 \%$ yield $)$. The composition was confirmed as $\mathrm{Zn}_{4} \mathrm{O}_{13} \mathrm{C}_{36} \mathrm{H}_{36}$ by elemental analysis: (\% calculated, found) $\mathrm{C}$ : 46.1, 46.1, H: 3.87, 3.86, Zn: 27.9, 25.4. The powder X-ray diffraction pattern (Bruker AXS D8 Advance diffractometer, Bragg-Brentano geometry, $\mathrm{Cu} \mathrm{K \alpha}$ radiation) of the porous form matches that calculated from the single crystal structure determination. The bands in the FTIR spectrum (Perkin Elmer Spectrum BX, KBr pellet, 4000-400 $\mathrm{cm}^{-1}$ ) are consistent with the organic module: 3428 (br), 2987 (m), 2953 (m), 2927 (m), 2867 (m), 1545 (s), 1443 (s), 1386 (m) $1369(\mathrm{~m}), 1234(\mathrm{~m}), 1051(\mathrm{w}), 1023(\mathrm{w}), 871(\mathrm{w}), 846(\mathrm{w}), 699(\mathrm{~m}), 516(\mathrm{~m}), 498(\mathrm{~m})$.

\section{Single Crystal X-ray Diffraction}

Single crystals of IRMOF-18 were prepared by heating a solution of 2,3,5,6tetramethylbenzene-1,4-dicarboxylic acid $(25 \mathrm{mg})$ and zinc nitrate hexahydrate $(500 \mathrm{mg})$ in $10 \mathrm{~mL}$ of $\mathrm{N}, \mathrm{N}$-diethylformamide in a tightly capped $20 \mathrm{~mL}$ scintillation vial at $100^{\circ} \mathrm{C}$ for 24 hr. A colourless block crystal $(0.38 \times 0.26 \times 0.18 \mathrm{~mm})$ was sealed in a capillary and analyzed at $293 \mathrm{~K}$ : cubic space group $F m-3 m$ with $a=25.6135(7) \AA, V=16803.8(8) \AA^{3}, Z=8$. Data were collected using graphite-monochromated Mo K $\alpha$ radiation on a Bruker SMART APEX diffractometer system. A total of 12108 reflections were collected in the range $2.25^{\circ}<\theta<$ $27.45^{\circ}$ of which 1014 were independent and 726 were observed $(I>2 \sigma[I])$. The Bruker SHELXTL program was used to solve the structure using "direct methods" techniques. All stages of weighted full-matrix least-squares refinement were conducted using $F_{\mathrm{o}}{ }^{2}$ data with the Bruker SHELXTL software package and converged to give $R_{1}=11.8 \%$ and $w R_{2}=35.7 \%$ $(I>2 \sigma[I])$. These values are typical for compounds of this type that contain large volume fractions of disordered solvent. Application of the SQUEEZE routine in the PLATON software package (Spek, A.L., Acta Cryst. A46, C-34 (1990)) reduced these values to $R_{1}=$ $7.3 \%$ and $w R_{2}=21.1 \%(I>2 \sigma[I]), S=1.08$. 


\section{Sorption Measurements}

A Cahn C-1000 microgravimetric balance was used to measure the change in mass of samples suspended within a glass enclosure under a chosen atmosphere. Samples (typically $400-600 \mathrm{mg}$ ) were cooled by a liquid nitrogen bath and the temperature was monitored using a thermocouple suspended in close proximity to the sample bucket. Prior to admittance of the analyte gas, the entire chamber and manifold were evacuated overnight while the walls were heated several times using a heat gun. The system was purged at room temperature three times with the analyte gas (ultra high purity grade) before cooling to $77 \mathrm{~K}$ and gases were passed through a molecular sieve trap before being exposed to the sample. When hydrogen gas was used, the trap was additionally immersed in liquid nitrogen to remove any condensable impurities. Pressures were measured with two MKS Baratron transducers 622A with the range covering 0-10 and 0-1000 Torr (accuracy 0.25\%). The adsorbate was added incrementally and data points were recorded when no further change in mass was observed (within $0.05 \mathrm{mg}$ ). An empirical buoyancy correction was applied to all data points based on the change in mass of standard aluminum foil weights within the analyte gas at $77 \mathrm{~K}$. Volumetric measurements were performed using a Micromeritics Co. sorption apparatus (Model 2010) with ultra high purity hydrogen gas. Evacuation and purging cycles were performed before all data collections.

Table S1. Gravimetric hydrogen sorption data for metal-organic frameworks at $77 \mathrm{~K}$.

\begin{tabular}{|c|c|c|c|c|c|c|c|c|c|}
\hline \multicolumn{2}{|c|}{ IRMOF-1 } & \multicolumn{2}{|c|}{ IRMOF-8 } & \multicolumn{2}{|c|}{ IRMOF-11 } & \multicolumn{2}{|c|}{ IRMOF-18 } & \multicolumn{2}{|c|}{ MOF-177 } \\
\hline $\begin{array}{c}\text { Pressure } \\
\text { (Torr) }\end{array}$ & $\begin{array}{l}\text { Uptake } \\
(\mathrm{mg} / \mathrm{g})\end{array}$ & $\begin{array}{c}\text { Pressure } \\
\text { (Torr) }\end{array}$ & $\begin{array}{l}\text { Uptake } \\
(\mathrm{mg} / \mathrm{g})\end{array}$ & $\begin{array}{c}\text { Pressure } \\
\text { (Torr) }\end{array}$ & $\begin{array}{l}\text { Uptake } \\
(\mathrm{mg} / \mathrm{g})\end{array}$ & $\begin{array}{c}\text { Pressure } \\
\text { (Torr) }\end{array}$ & $\begin{array}{l}\text { Uptake } \\
(\mathrm{mg} / \mathrm{g})\end{array}$ & $\begin{array}{c}\text { Pressure } \\
\text { (Torr) }\end{array}$ & $\begin{array}{l}\text { Uptake } \\
(\mathrm{mg} / \mathrm{g})\end{array}$ \\
\hline 0.0 & 0.00 & 0.0 & 0.00 & 0.0 & 0.00 & 0.0 & 0.00 & 0.0 & 0.00 \\
\hline 10.9 & 0.38 & 9.2 & 1.67 & 4.8 & 1.05 & 13.7 & 0.19 & 9.8 & 0.26 \\
\hline 20.7 & 0.75 & 45.0 & 3.78 & 9.5 & 1.69 & 33.1 & 0.65 & 69.1 & 1.72 \\
\hline 48.4 & 1.69 & 98.0 & 5.54 & 21.0 & 2.48 & 60.5 & 1.19 & 141.2 & 3.32 \\
\hline 77.4 & 2.53 & 150.8 & 6.97 & 38.0 & 3.31 & 101.3 & 1.92 & 240.7 & 5.13 \\
\hline 100.4 & 3.13 & 200.2 & 8.02 & 53.7 & 4.02 & 199.2 & 3.40 & 298.8 & 6.17 \\
\hline 142.3 & 4.20 & 250.1 & 8.98 & 99.5 & 5.56 & 297.9 & 4.62 & 400.0 & 7.76 \\
\hline 149.9 & 4.33 & 295.8 & 9.79 & 149.3 & 7.01 & 402.7 & 5.83 & 499.0 & 9.18 \\
\hline 201.1 & 5.43 & 348.6 & 10.61 & 200.0 & 8.17 & 501.0 & 6.73 & 592.0 & 10.40 \\
\hline 251.4 & 6.36 & 402.4 & 11.33 & 252.2 & 9.34 & 598.6 & 7.63 & 702.0 & 11.85 \\
\hline 301.9 & 7.27 & 501.2 & 12.52 & 298.6 & 10.26 & 753.0 & 8.89 & 751.0 & 12.45 \\
\hline 357.1 & 8.16 & 605.0 & 13.60 & 401.9 & 11.88 & 697.6 & 8.50 & 651.7 & 11.27 \\
\hline 401.4 & 8.84 & 733.6 & 14.79 & 502.5 & 13.27 & 551.1 & 7.28 & 455.0 & 8.63 \\
\hline 451.0 & 9.60 & 755.3 & 14.97 & 602.2 & 14.46 & 352.7 & 5.43 & 198.0 & 4.50 \\
\hline 500.9 & 10.23 & 352.1 & 10.59 & 703.7 & 15.65 & 150.7 & 2.81 & & \\
\hline 599.1 & 11.49 & 77.0 & 4.95 & 753.2 & 16.19 & 44.3 & 1.01 & & \\
\hline 702.1 & 12.70 & 29.8 & 3.07 & 552.4 & 14.02 & & & & \\
\hline 752.9 & 13.24 & 10.1 & 1.72 & 351.0 & 11.12 & & & & \\
\hline 558.8 & 11.00 & 3.4 & 0.86 & 221.9 & 8.83 & & & & \\
\hline 449.5 & 9.52 & & & 124.3 & 6.54 & & & & \\
\hline 277.4 & 6.86 & & & 75.1 & 4.88 & & & & \\
\hline 124.2 & 3.72 & & & 39.6 & 3.73 & & & & \\
\hline 62.9 & 2.16 & & & 5.8 & 1.56 & & & & \\
\hline 30.8 & 1.09 & & & 1.9 & 0.93 & & & & \\
\hline 0.014 & 0.00 & & & & & & & & \\
\hline
\end{tabular}


Table S2. Volumetric hydrogen sorption data for metal-organic frameworks at $77 \mathrm{~K}$.

\begin{tabular}{|cc|cc|cc|cc|cc|}
\hline \multicolumn{2}{|c|}{ IRMOF-1 } & \multicolumn{2}{c|}{ IRMOF-8 } & \multicolumn{2}{c|}{ IRMOF-11 } & \multicolumn{2}{c|}{ IRMOF-18 } & \multicolumn{2}{c|}{ MOF-177 } \\
\hline $\begin{array}{c}\text { Pressure } \\
(\text { Torr })\end{array}$ & $\begin{array}{c}\text { Uptake } \\
\left(\mathrm{cm}_{\text {STP }}^{3} / \mathrm{g}\right)\end{array}$ & $\begin{array}{c}\text { Pressure } \\
(\text { Torr })\end{array}$ & $\begin{array}{c}\text { Uptake } \\
\left(\mathrm{cm}_{\text {STP }}^{3} \text { /g) }\right.\end{array}$ & $\begin{array}{c}\text { Pressure } \\
(\text { Torr })\end{array}$ & $\begin{array}{c}\text { Uptake } \\
\left(\mathrm{cm}^{3}{ }_{\text {STP }} / \mathrm{g}\right)\end{array}$ & $\begin{array}{c}\text { Pressure } \\
(\text { Torr })\end{array}$ & $\begin{array}{c}\text { Uptake } \\
\left(\mathrm{cm}_{\text {STP }}^{3} / \mathrm{g}\right)\end{array}$ & $\begin{array}{c}\text { Pressure } \\
(\text { Torr })\end{array}$ & $\begin{array}{c}\text { Uptake } \\
\left(\mathrm{cm}^{3} \text { STP }^{3} / \mathrm{g}\right)\end{array}$ \\
\hline 0.147 & 0.120 & 0.091 & 0.331 & 0.086 & 0.5754 & 0.104 & 0.04 & 0.136 & 0.0721 \\
0.901 & 0.746 & 0.862 & 3.58 & 0.862 & 5.384 & 0.870 & 0.360 & 0.902 & 0.5226 \\
10.9 & 6.70 & 10.0 & 22.06 & 11.0 & 26.38 & 9.1 & 2.89 & 9.2 & 4.26 \\
13.9 & 8.10 & 13.0 & 25.40 & 13.6 & 29.05 & 13.1 & 3.92 & 14.2 & 6.20 \\
20.2 & 11.04 & 17.5 & 29.59 & 17.6 & 32.59 & 17.9 & 5.09 & 17.7 & 7.41 \\
27.4 & 14.02 & 25.9 & 35.88 & 26.0 & 38.56 & 27.2 & 7.23 & 26.8 & 10.43 \\
43.3 & 20.15 & 43.5 & 46.18 & 44.0 & 48.73 & 45.6 & 11.10 & 43.2 & 15.31 \\
69.5 & 29.12 & 68.9 & 57.75 & 70.0 & 60.33 & 70.2 & 15.92 & 71.1 & 22.80 \\
86.8 & 34.48 & 86.5 & 64.36 & 86.4 & 66.73 & 86.7 & 18.96 & 87.6 & 26.92 \\
264.2 & 74.76 & 261.1 & 107.51 & 265.4 & 114.69 & 259.9 & 44.89 & 265.5 & 62.08 \\
430.3 & 99.26 & 431.9 & 133.69 & 431.2 & 143.14 & 432.5 & 63.85 & 431.2 & 86.90 \\
516.3 & 108.73 & 516.3 & 143.72 & 516.4 & 154.51 & 516.0 & 71.31 & 516.3 & 97.64 \\
757.2 & 126.81 & 756.8 & 165.86 & 757.2 & 178.72 & 756.8 & 88.03 & 757.1 & 122.56 \\
\hline
\end{tabular}

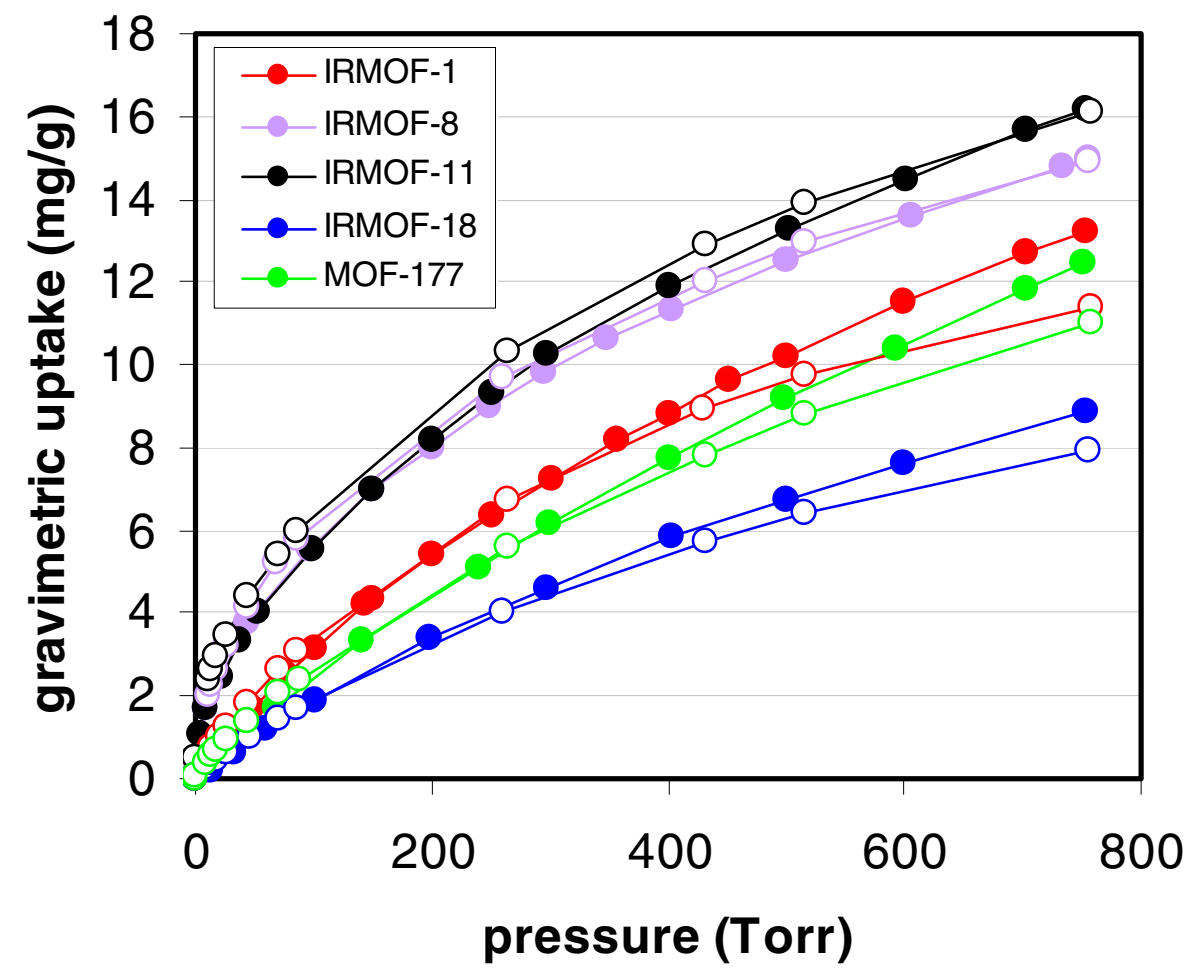

Figure S1. Hydrogen adsorption isotherms for metal-organic frameworks measured gravimetrically (solid markers) and volumetrically (open markers) at $77 \mathrm{~K}$. The volumetric data have been converted by the factor $0.090 \mathrm{mg}_{\mathrm{H} 2} / \mathrm{cm}^{3}(\mathrm{STP})$. 


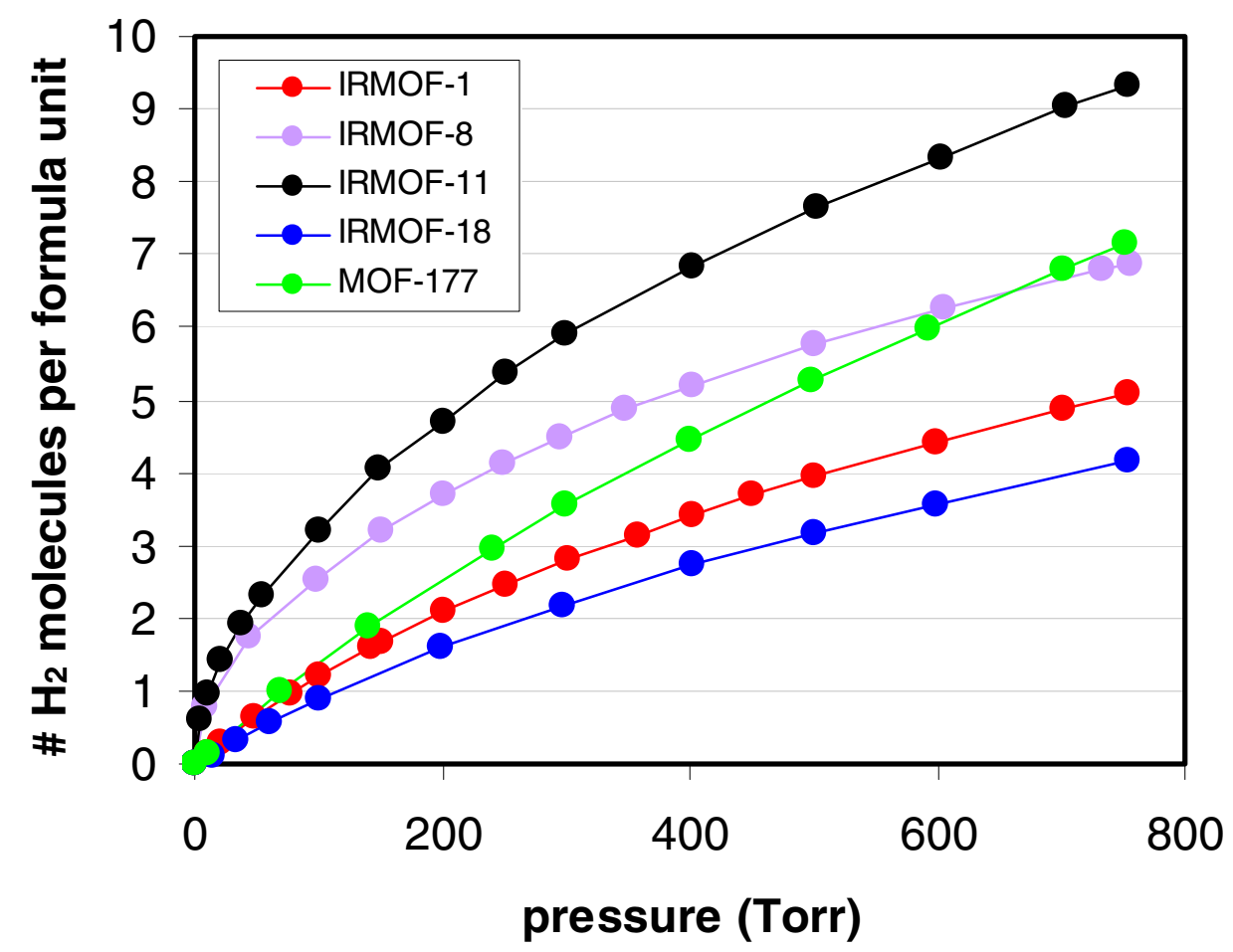

Figure S2. Hydrogen adsorption isotherms normalized to a molar scale.

\section{Calculations of the Accessible and Hydrogen-Occupied Volumes}

The accessible crystal volume $V_{a c c}$ of each material was calculated with the Cerius ${ }^{2}$ software package (Accelrys) using the appropriate van der Waals radii and a probe radius of $1.45 \AA$. The volume occupied by molecular hydrogen (at $1 \mathrm{~atm}$ and $77 \mathrm{~K}$ ) $V_{o c c}$ was calculated by

$$
V_{o c c}=Z \times N \times V_{H 2}
$$

where $Z$ is the number of formula units per unit cell, $N$ is the number of $\mathrm{H}_{2}$ molecules adsorbed per formula unit, and $V_{H 2}$ is the kinetic volume of a hydrogen molecule, calculated using a kinetic diameter of $2.89 \AA$ A (from D.W. Breck, Zeolite Molecular Sieves, Kreiger: Malabar, 1974).

Table S3. Accessible and hydrogen-occupied volume fractions of metal-organic frameworks at $1 \mathrm{~atm}$ and $77 \mathrm{~K}$.

\begin{tabular}{ccccc}
\hline Material & $\begin{array}{c}\boldsymbol{V}_{\text {acc }} \\
\left(\mathbf{1 0} \mathbf{\AA}^{\mathbf{3}} / \text { cell }\right)\end{array}$ & $\begin{array}{c}\text { accessible fraction, } \\
\boldsymbol{V}_{\text {acc }} / \boldsymbol{V}_{\text {cell }}\end{array}$ & $\begin{array}{c}\boldsymbol{V}_{\text {occ }} \\
\left(\AA^{\mathbf{3}} / \text { cell }\right)\end{array}$ & $\begin{array}{c}\text { occupied fraction, } \\
\boldsymbol{V}_{\text {occ }} / \boldsymbol{V}_{\text {acc }}\end{array}$ \\
\hline IRMOF-1 & 1.02 & 0.59 & 506 & 0.050 \\
IRMOF-8 & 1.79 & 0.66 & 698 & 0.039 \\
IRMOF-11 & 1.23 & 0.40 & 1411 & 0.115 \\
IRMOF-18 & 0.701 & 0.42 & 425 & 0.061 \\
MOF-177 & 2.25 & 0.63 & 718 & 0.032 \\
\hline
\end{tabular}

